\title{
ON THE BEHAVIOR OF THE CONSTANT IN A DECOUPLING INEQUALITY FOR MARTINGALES
}

\author{
PAWEL HITCZENKO
}

(Communicated by Lawrence F. Gray)

ABstract. Let $\left(f_{n}\right)$ and $\left(g_{n}\right)$ be two martingales with respect to the same filtration $\left(\mathscr{F}_{n}\right)$ such that their difference sequences $\left(d_{n}\right)$ and $\left(e_{n}\right)$ satisfy

$$
P\left(d_{n} \geq \lambda \mid \mathscr{F}_{n-1}\right)=P\left(e_{n} \geq \lambda \mid \mathscr{F}_{n-1}\right)
$$

for all real $\lambda$ 's and $n \geq 1$. It is known that

$$
\left\|f^{*}\right\|_{p} \leq K_{p}\left\|g^{*}\right\|_{p}, \quad 1 \leq p<\infty,
$$

for some constant $K_{p}$ depending only on $p$. We show that $K_{p}=O(p)$. This will be obtained via a new version of Rosenthal's inequality which generalizes a result of Pinelis and which may be of independent interest.

\section{INTRODUCTION AND STATEMENT OF RESULTS}

Let us begin with some notation. Given an increasing sequence of $\sigma$-algebras $\left(\mathscr{F}_{n}\right)$ on some probability space $(\Omega, \mathscr{F}, P)$, we denote by $E_{n-1}(\cdot)=E\left(\cdot \mid \mathscr{F}_{n-1}\right)$ the conditional expectation operator (with the convention that $\mathscr{F}=\{\varnothing, \Omega\}$ and $E_{0}=E$, the expectation operator). The $L_{p}$-norm of a random variable $X$ is denoted $\|X\|_{p}, 1 \leq p \leq \infty$. A sequence $\left(f_{n}\right)$ of integrable random variables is a martingale if it is $\left(\mathscr{F}_{n}\right)$-adapted (i.e., $f_{n}$ is $\mathscr{F}_{n}$-measurable, $n \geq 1$ ) and $E_{n-1} f_{n}=f_{n-1}, n \geq 1$. Given a martingale $\left(f_{n}\right)$, its difference sequence $\left(d_{n}\right)$ is defined by $d_{n}=f_{n}-f_{n-1}, n \geq 0$, and we will write

$$
s(f)=\left(\sum_{k=1}^{\infty} E_{k-1} d_{k}^{2}\right)^{1 / 2} \text { and } s_{n}(f)=\left(\sum_{k=1}^{n} E_{k-1} d_{k}^{2}\right)^{1 / 2} .
$$

For any sequence $\left(X_{n}\right)$ of random variables $X^{*}$ denotes $\sup _{n \geq 1}\left|X_{n}\right|$ and

$$
X_{n}^{*}=\max _{1 \leq k \leq n}\left|X_{k}\right| \text {. }
$$

The indicator function of a set $A$ is denoted by $I(A)$. Let us recall that two $\left(\mathscr{F}_{n}\right)$-adapted sequences of random variables $\left(X_{n}\right)$ and $\left(Y_{n}\right)$ are tangent if for each real number $\lambda$ one has

$$
P\left(X_{n} \geq \lambda \mid \mathscr{F}_{n-1}\right)=P\left(Y_{n} \geq \lambda \mid \mathscr{F}_{n-1}\right) \quad \text { a.s., } n=1,2, \ldots
$$

Received by the editors September 6, 1991 and, in revised form, August 17, 1992.

1991 Mathematics Subject Classification. Primary 60E15, 60G42.

Key words and phrases. Moment inequalities, martingale, tangent sequences.

This research was supported in part by the NSF grant DMS-8921369. 
Let now $\left(d_{n}\right)$ and $\left(e_{n}\right)$ be two tangent sequences of martingale differences and denote by $\left(f_{n}\right)$ and $\left(g_{n}\right)$ the corresponding martingales. Then the following inequality holds:

$$
\left\|f^{*}\right\|_{p} \leq K_{p}\left\|g^{*}\right\|_{p}, \quad 1 \leq p<\infty,
$$

where $K_{p}$ is a constant depending only on $p$. At least three different proofs are available (cf. $[3,6,11]$ and references therein). Our aim here is to identify asymptotic behavior of the constant $K_{p}$ as a function of $p$. Namely, we have

Theorem 1. Let $\left(f_{n}\right)$ and $\left(g_{n}\right)$ be two martingales with tangent difference sequences. Then (1) holds with $K_{p}=O(p)$.

(For a companion result for nonnegative random variables see Hitczenko [4].) It is probably worth noticing here that $K_{p} \geq O(p)$. To see this, one just needs to observe that if $\left(h_{n}\right)$ is a Haar system and $\left(\varepsilon_{n}\right)$ is an arbitrary sequence of signs then $\left(h_{n}\right)$ and $\left(\varepsilon_{n} h_{n}\right)$ are tangent sequences of martingale differences and then use the well-known fact that unconditional constant of a Haar system in $L_{p}$ is of order $p, 1<p<\infty$. Thus, the upper bound on $K_{p}$ is what is new.

The simplest way to justify (1) (at least for $p \geq 2$ ) is via Rosenthal's inequality:

$$
\begin{aligned}
A_{p}^{-1} & \left\{\|s(f)\|_{p}+\left(\sum\left\|d_{k}\right\|_{p}^{p}\right)^{1 / p}\right\} \\
& \leq\left\|f^{*}\right\|_{p} \leq B_{p}\left\{\|s(f)\|_{p}+\left(\sum\left\|d_{k}\right\|_{p}^{p}\right)^{1 / p}\right\},
\end{aligned}
$$

valid for all martingales $\left(f_{n}\right)$ and $2 \leq p<\infty$. Given (2), (1) follows with $K_{p} \leq$ $A_{p} \cdot B_{p}$, since for all $0<r<\infty$ and $n \geq 1$ we have $E_{n-1}\left|d_{n}\right|^{r}=E_{n-1}\left|e_{n}\right|^{r}$. But $A_{p}=O(\sqrt{p})$ (cf., e.g., [2, Theorem IV, 3.1]) and $B_{p}=O(p / \ln p$ ) (Hitczenko [5]), which gives $K_{p} \leq O\left(p^{3 / 2} / \ln p\right)$, a bound far too big for our purposes. On the other hand, since the two terms appearing in Rosenthal's inequality are usually of different size, one may want to have different constants attached to those two terms. As was pointed out to us by Pinelis, several results in that direction were published in the Russian literature. For example, assuming that $\left(d_{n}\right)$ is a sequence of independent mean zero random variables, Sazonov [10] showed that

$$
\left\|\sum d_{k}\right\|_{p} \leq K\left\{2^{p / 4}\left(\sum\left\|d_{k}\right\|_{2}^{2}\right)^{1 / 2}+\left(\sum\left\|d_{k}\right\|_{p}^{p}\right)^{1 / p}\right\},
$$

while Nagaev and Pinelis [8] proved

$$
\left\|\sum d_{k}\right\|_{p} \leq K\left\{\sqrt{p}\left(\sum\left\|d_{k}\right\|_{2}^{2}\right)^{1 / 2}+p\left(\sum\left\|d_{k}\right\|_{p}^{p}\right)^{1 / p}\right\} .
$$

The latter inequality was generalized to martingales as follows (Pinelis [9]): suppose $\left(d_{n}\right)$ is a martingale difference sequence such that $\left\|E_{n-1} d_{n}^{2}\right\|_{\infty}<\infty$ for all $n \geq 1$. Then

$$
\left\|\sum d_{k}\right\|_{p} \leq K\left\{\sqrt{p}\left(\sum\left\|E_{k-1} d_{k}^{2}\right\|_{\infty}\right)^{1 / 2}+p\left(\sum\left\|d_{k}\right\|_{p}^{p}\right)^{1 / p}\right\} .
$$

We will extend this result to 
Theorem 2. There exists an absolute constant $B$ such that for every $p, 2 \leq p<$ $\infty$, and every martingale $\left(f_{n}, \mathscr{F}_{n}\right)$ with difference sequence $\left(d_{n}\right)$ one has

$$
\left\|f^{*}\right\|_{p} \leq B\left\{\sqrt{p}\|s(f)\|_{p}+p\left\|d^{*}\right\|_{p}\right\} .
$$

Theorem 2 is exactly what is needed to conclude Theorem 1 . This is simply because the following inequalities are true:

$$
\left\|d^{*}\right\|_{p} \leq 2^{1 / p}\left\|e^{*}\right\|_{p} \leq 2^{(p+1) / p}\left\|g^{*}\right\|_{p}
$$

and

$$
\|s(f)\|_{p}=\|s(g)\|_{p} \leq \sqrt{p / 2}\left\|g^{*}\right\|_{p}, \quad 2 \leq p<\infty .
$$

The first inequality follows from Hitczenko [3, Lemma 1] while the second can be found, e.g., in Garsia [2, Theorem IV 3.1]. It remains to justify Theorem 2, which we will do in the next section.

\section{Proof of Theorem 2}

Our approach will closely follow the proof given in Hitczenko [5]; it is based on a powerful "good- $\lambda$ inequality" technique of Burkholder [1] combined with exponential estimates for martingales. The main difference is that, rather than stopping sequences $\left(d_{n}\right)$ and $\left(s_{n}(f)\right)$ on the same level (as was done in Hitczenko [5]), we will stop them on different levels. This gives more freedom in choosing various parameters involved in the good- $\lambda$ inequality, allowing us to attach constants of different size to each of the two terms on the right-hand side of Rosenthal's inequality. Our departing point is, as in Hitczenko [5], the following extension of Prokhorov's "arcsinh" inequality (also proved independently by Leventhal [7]).

Lemma. Let $\left(f_{n}\right)$ be a martingale such that $\left\|d^{*}\right\|_{\infty}=M<\infty$ and $\left\|s^{2}(f)\right\|_{\infty}=$ $K^{2}<\infty$. Then for every positive number $\lambda$ one has

$$
P\left(\left|f_{n}\right|>\lambda\right) \leq 2 \exp \left\{-\frac{\lambda}{2 M} \operatorname{arcsinh} \frac{\lambda M}{2 K^{2}}\right\} .
$$

Let now $\left(d_{n}\right)$ be a martingale difference sequence and assume first that there exists a sequence $\left(w_{n}\right)$ such that $\left|d_{n}\right| \leq w_{n}$ and $w_{n}$ is $\mathscr{F}_{n-1}$ measurable, for $n=1,2, \ldots$. Let $\beta, \delta_{1}, \delta_{2}$ be positive numbers chosen so that $0<\delta_{i}<\beta-1$, $i=1,2$. Since for all measurable sets $A, B$, and $C$,

$$
\begin{aligned}
P(A) & =P(A \cap(B \cup C))+P\left(A \cap B^{c} \cap C^{c}\right) \\
& \leq P(B)+P(C)+P\left(A \cap B^{c} \cap C^{c}\right),
\end{aligned}
$$

for all positive $\lambda$ 's one has

$$
\begin{aligned}
P\left(f^{*} \geq \beta \lambda\right) \leq & P\left(d^{*} \geq \delta_{1} \lambda\right)+P\left(s(f) \geq \delta_{2} \lambda\right) \\
& +P\left(f^{*} \geq \beta \lambda, d^{*}<\delta_{1} \lambda, s(f)<\delta_{2} \lambda\right) .
\end{aligned}
$$

To estimate the last probability on the right-hand side let us define the following stopping times:

$$
\begin{aligned}
\nu & =\inf \left\{n:\left|f_{n}\right| \geq \beta \lambda\right\}, & \mu & =\inf \left\{n:\left|f_{n}\right| \geq \lambda\right\}, \\
\tau_{1} & =\inf \left\{n: w_{n+1} \geq \delta_{1} \lambda\right\}, & \tau_{2} & =\inf \left\{n: s_{n+1}(f) \geq \delta_{2} \lambda\right\},
\end{aligned}
$$


and let $\tau=\tau_{1} \wedge \tau_{2}$. Then, following usual computation (cf. Burkholder [1]), we obtain that

$$
\begin{aligned}
P\left(f^{*}\right. & \left.\geq \beta \lambda, d^{*}<\delta_{1} \lambda, s(f)<\delta_{2} \lambda\right)=P(\nu<\infty, \tau=\infty) \\
& \leq P\left(\left|\sum_{k=\mu}^{\nu \wedge \tau} d_{k}\right| \geq\left(\beta-1-\delta_{1}\right) \lambda\right) \\
& =E P\left(\left|\sum_{k=\mu}^{\nu \wedge \tau} d_{k}\right| \geq\left(\beta-1-\delta_{1}\right) \lambda \mid \mathscr{F}_{\mu}\right) \\
& \leq 2 E \exp \left\{-\frac{\left(\beta-1-\delta_{1}\right) \lambda}{2 \delta_{1} \lambda} \operatorname{arcsinh} \frac{\left(\beta-1-\delta_{1}\right) \delta_{1} \lambda^{2}}{2 \delta_{2}^{2} \lambda^{2}}\right\} I(\mu<\infty) \\
& =2 E \exp \left\{-\frac{\beta-1-\delta_{1}}{2 \delta_{1}} \operatorname{arcsinh} \frac{\left(\beta-1-\delta_{1}\right) \delta_{1}}{2 \delta_{2}^{2}}\right\} P(\mu<\infty) .
\end{aligned}
$$

Suppose for a moment that $\beta, \delta_{1}$, and $\delta_{2}$, all depending on $p$, are chosen so that $\left(\beta-1-\delta_{1}\right) \delta_{1} / 2 \delta_{2}^{2}$ remains bounded as a function of $p$. Let $A$ be any number satisfying $\left(\beta-1-\delta_{1}\right) \delta_{1} / 2 \delta_{2}^{2} \leq A$. Then, using the fact that the function $x^{-1} \operatorname{arcsinh} x$ is decreasing for $x>0$ we infer that there exists a constant $C$ (which may actually be chosen to be $A^{-1} \operatorname{arcsinh} A$ ) such that

$$
\exp \left\{-\frac{\beta-1-\delta_{1}}{2 \delta_{1}} \operatorname{arcsinh} \frac{\left(\beta-1-\delta_{1}\right) \delta_{1}}{2 \delta_{2}^{2}}\right\} \leq \exp \left\{-C \frac{\left(\beta-1-\delta_{1}\right)^{2}}{2 \delta_{2}^{2}}\right\} .
$$

Therefore,

$$
\begin{aligned}
P\left(f^{*} \geq \beta \lambda\right) \leq & P\left(d^{*} \geq \delta_{1} \lambda\right)+P\left(s(f) \geq \delta_{2} \lambda\right) \\
& +2 \exp \left\{-C \frac{\left(\beta-1-\delta_{1}\right)^{2}}{2 \delta_{2}^{2}}\right\} P\left(f^{*} \geq \lambda\right),
\end{aligned}
$$

which, after integration, yields

$$
\frac{1}{\beta^{p}}\left\|f^{*}\right\|_{p}^{p} \leq \frac{1}{\delta_{1}^{p}}\left\|d^{*}\right\|_{p}^{p}+\frac{1}{\delta_{2}^{p}}\|s(f)\|_{p}^{p}+2 \exp \left\{-C \frac{\left(\beta-1-\delta_{1}\right)^{2}}{2 \delta_{2}^{2}}\right\}\left\|f^{*}\right\|_{p}^{p} .
$$

This, in turn, implies that

$$
\left\|f^{*}\right\|_{p}^{p} \leq L^{-1}\left\{\left(\frac{\beta}{\delta_{1}}\right)^{p}\left\|d^{*}\right\|_{p}^{p}+\left(\frac{\beta}{\delta_{2}}\right)^{p}\|s(f)\|_{p}^{p}\right\}
$$

as long as

$$
L=1-2 \beta^{p} \exp \left\{-C \frac{\left(\beta-1-\delta_{1}\right)^{2}}{2 \delta_{2}^{2}}\right\}
$$

is positive and bounded away from zero. So, in order to complete our proof, it remains to choose $\beta, \delta_{1}$, and $\delta_{2}$ so that all of the above requirements are satisfied and

$$
L^{-1}\left(\beta / \delta_{1}\right)^{p} \leq B^{p} p^{p} \quad \text { and } \quad L^{-1}\left(\beta / \delta_{2}\right)^{p} \leq B^{p} \sqrt{p^{p}},
$$

for some absolute constant $B$. If we now put $\beta=2, \delta_{1}=\eta_{1} / p, \delta_{2}=\eta_{2} / \sqrt{p}$ with $0<\eta_{i}<1, i=1,2$; then obviously $0<\delta_{i}<\beta-1$, and we have $\left(\beta / \delta_{1}\right)^{p}=\left(2 / \eta_{1}\right)^{p} p^{p},\left(\beta / \delta_{2}\right)^{p}=\left(2 / \eta_{2}\right)^{p} \sqrt{p^{p}}$. To see that $L$ is bounded away 
from zero we need to bound $\beta^{p} \exp \left\{-C\left(\beta-1-\delta_{1}\right)^{2} / 2 \delta_{2}^{2}\right\}$ from above by a number strictly smaller than $1 / 2$. But

$$
\begin{gathered}
\beta^{p} \exp \left\{-C \frac{\left(\beta-1-\delta_{1}\right)^{2}}{2 \delta_{2}^{2}}\right\} \leq \exp \left\{p\left(1-C \frac{\left(1-\eta_{1} / p\right)^{2}}{2 \eta_{2}^{2}}\right)\right\} \\
\leq \exp \left\{p\left(1-\frac{C}{8 \eta_{2}^{2}}\right)\right\} \leq \exp \{-p\},
\end{gathered}
$$

provided $C>16 \eta_{2}^{2}$. Since $C$ may be taken to be

$$
\begin{aligned}
\frac{2 \delta_{2}^{2}}{\left(\beta-1-\delta_{1}\right) \delta_{1}} \operatorname{arcsinh} \frac{\left(\beta-1-\delta_{1}\right) \delta_{1}}{2 \delta_{2}^{2}} & =\frac{2 \eta_{2}^{2}}{\left(1-\eta_{1} / p\right) \eta_{1}} \operatorname{arcsinh} \frac{\left(1-\eta_{1} / p\right) \eta_{1}}{2 \eta_{2}^{2}} \\
& \geq \frac{2 \eta_{2}^{2}}{\eta_{1}} \operatorname{arcsinh} \frac{\eta_{1}}{2 \eta_{2}^{2}},
\end{aligned}
$$

it suffices to choose $\eta_{i}$ 's so that

$$
\frac{2 \eta_{2}^{2}}{\eta_{1}} \operatorname{arcsinh} \frac{\eta_{1}}{2 \eta_{2}^{2}} \geq 16 \eta_{2}^{2} \text { or } \operatorname{arcsinh} \frac{\eta_{1}}{2 \eta_{2}^{2}} \geq 8 \eta_{1} \text {, }
$$

which, of course, is possible. This completes the first part of the proof.

The general case can be reduced to this special one via Davis' decomposition: given a martingale difference sequence $\left(d_{n}\right)$ one writes

$$
\begin{aligned}
d_{n}= & a_{n}+b_{n} \\
= & d_{n} I\left(\left|d_{n}\right| \leq 2 d_{n-1}^{*}\right)-E_{n-1} d_{n} I\left(\left|d_{n}\right| \leq 2 d_{n-1}^{*}\right) \\
& +d_{n} I\left(\left|d_{n}\right|>2 d_{n-1}^{*}\right)-E_{n-1} d_{n} I\left(\left|d_{n}\right|>2 d_{n-1}^{*}\right) .
\end{aligned}
$$

Then $\left|a_{n}\right|$ is bounded by $\mathscr{F}_{n-1}$ measurable random variable $4 d_{n-1}^{*}$ and, since $\left|d_{k}\right|<2\left(d_{k}^{*}-d_{k-1}^{*}\right)$, when $\left|d_{k}\right|>2 d_{k-1}^{*}$

$$
\sum\left|d_{k}\right| I\left(\left|d_{k}\right|>2 d_{k-1}^{*}\right) \leq 2 d^{*}
$$

Therefore, the $L_{p}$-norm of the maximal function of the second martingale is controlled by

$$
\left\|\sum\left|b_{k}\right|\right\|_{p} \leq 2\left\|d^{*}\right\|_{p}+\left\|\sum E_{k-1}\left|d_{k}\right| I\left(d_{n} \mid>2 d_{n-1}^{*}\right)\right\|_{p} \leq(2+2 p)\left\|d^{*}\right\|_{p},
$$

by the convex function inequality. The proof is completed.

\section{ACKNOWLEDGMENTS}

I would like to thank Iosif Pinelis for bringing his paper [9] to my attention.

\section{REFERENCES}

1. D. L. Burkholder, Distribution function inequalities for martingales, Ann. Probab. 1 (1973), 19-42.

2. A. M. Garsia, Martingale inequalities, Seminar Notes on Recent Progress, Benjamin, Reading, MA, 1973.

3. P. Hitczenko, Comparison of moments for tangent sequences of random variables, Probab. Theory Related Fields 78 (1988), 223-230.

4. _ Best constant in the decoupling inequality for non-negative random variables, Statist. Probab. Letters 9 (1990), 327-329. 
5. (1990), 1656-1668.

6. S. Kwapień and W. A. Woyczyński, Tangent sequences of random variables: basic inequalities and their applications, Proc. Conf. on Almost Everywhere Convergence in Probability and Ergodic Theory (Columbus, OH, 1988) (G. Edgar and L. Sucheston, eds.), Academic Press, San Diego, CA, 1989, pp. 237-265.

7. S. Leventhal, $A$ uniform CLT for uniformly bounded families of martingale differences, J. Theoret. Probab. 2 (1989), 271-287.

8. S. V. Nagaev and I. F. Pinelis, Some inequalities for the distributions of sums of independent random variables, Theory Probab. Appl. 22 (1977), 248-256.

9. I. F. Pinelis, Estimates of moments of infinite-dimensional martingales, Math. Notes 27 (1980), 459-462.

10. V. V. Sazonov, On the estimation of moments of sums of independent random variables, Theory Probab. Appl. 19 (1974), 371-374.

11. J. Zinn, Comparison of martingale differences, Probability in Banach Spaces V (A. Beck, R. Dudley, M. Hahn, J. Kuelbs, and M. Marcus, eds.), Lecture Notes in Math., vol. 1153, Springer-Verlag, Berlin and New York, 1985, pp. 453-457.

Department of Mathematics, North Carolina State University, Raleigh, North CarOLINA 27695-8205

E-mail address: pawe1@math.ncsu.edu 